\title{
Applying Knowledge in New Contexts: A Comparison of Pre- and Post-Instruction Students
}

\author{
Dyan L. McBride ${ }^{1}$ and Dean A. Zollman \\ Kansas State University, Manhattan KS, 66502 \\ ${ }^{1}$ Present Address: Mercyhurst College, Erie PA, 16509
}

\begin{abstract}
This study focuses on how students apply previous learning of light and basic geometric optics to the context of wavefront aberrometry. In one aspect of this study we compared the application of previous learning of students who had studied light and basic geometric optics in a physics course with those who had not and thus could only apply knowledge obtained in an informal way. We sought to examine what differences exist in the way they construct an understanding of wavefront aberrometry. The data showed that students with no formal instruction tended to rely on experiential knowledge as one would expect. However, the students with formal instruction relied on textbook knowledge and tended to discount or ignore their everyday experiences. We will discuss what this difference in knowledge types might imply about the knowledge construction process.
\end{abstract}

Keywords: Physics education, prior knowledge, transfer, resources

PACS: $01.40 . \mathrm{Fk}$

\section{INTRODUCTION}

For the past several years, we have undertaken a series of studies to investigate how students apply their knowledge or learning in physics to understand phenomena that they have not previously seen. One such investigation looked at how students apply their knowledge of light and basic geometric optics to the new context of wavefront aberrometry. Wavefront aberrometry diagnoses vision defects by utilizing the optical properties of light instead of the subjective judgment of patients $[1,2]$. It is relatively new to ophthalmology and thus provides a good context for this type of research.

Recently we examined the resources that two groups of students use as they constructed an understanding of the physics of wavefront aberrometry. One group had completed formal university instruction in geometrical and physical optics (called the post-instruction students) while the other group had not (pre-instruction). One aspect of the investigation was to address the research question: What are the differences, if any, in the resources used by pre- and post-instruction students and the ways in which they use prior knowledge when constructing an understanding of this new context?

\section{LITERATURE REVIEW}

Much work has been conducted regarding students' understanding of light. These efforts include students' views on the flux concept of light [3], the application of light in real world situations [4], and light propagation [5].

A large body of work also exists on student understanding of lenses and geometric optics. In studies by Goldberg and McDermott, students were found to have difficulty understanding image formation by a plane mirror [6] and converging lenses [7]. Galili et al. found a similar result, calling students' conceptualization of image formation a "projected-image conceptualization ... a hybridization of their pre-instruction holistic conceptualization and the formal physics conceptualization [8]."

\section{METHODOLOGY}

The research was conducted through individual learning/teaching interviews [9] with students who were both pre- and post-instruction in light and basic optics. The data sources for this study consist of the video and audio recordings of the interviews, full transcriptions, student sketches and field notes, all of 
which were analyzed with an overarching phenomenographic approach [10].

During each interview of approximately 45 minutes, students were encouraged to think-aloud as they responded and to explain their reasoning for all answers. For details about the model aberrometer, interview protocol, and procedures, see [11].

In agreement with our previous work, the data were analyzed in such a way to identify the resources students utilize when constructing an understanding of the new context of wavefront aberrometry. Resources can be thought of as the fragments of information, knowledge, and experience that individuals bring to a new situation or context [12]. In this perspective, resources are universal and encompass all knowledge, and are classified as neither correct nor incorrect.

\section{Student Demographics}

The pre-instruction students were enrolled in the first-semester of introductory-level algebra-based physics, but had not yet received any formal instruction on the topics discussed here.

The study was also conducted with post-instruction students. This group was enrolled in the second semester of an introductory-level algebra-based physics course at Kansas State University. The course is a traditional lecture-recitation-laboratory class. At the time of the interviews, students had completed their entire unit on light and optics including lectures, recitations, textbook homework problems, and an exam. The coverage included the topics of light, mirrors, lenses, and basic information about the human eye such as the optics of near- and farsightedness.

It should be noted that a second group of students was also interviewed. These students completed a research-based learning activity on optics of the human eye that was constructed by us and included both hands-on and computer visualization activities. Given the length constraint of this paper, we will focus on only the pre-instruction and traditional postinstruction groups.

\section{RESULTS AND DISCUSSION}

To compare the pre-instruction and post-instruction students, four aspects of the knowledge construction process were considered: basic knowledge about vision, activated resources for understanding wavefront aberrometry, approach to knowledge construction, and the scaffolding which we needed to supply for the students to reach that understanding.

\section{Prior Knowledge about Vision}

This study found that both pre- and post-instruction students have similar prior knowledge about vision and the human eye. First and foremost, nearly all students have an incomplete and self-inconsistent understanding of how the human eye functions. Both pre- and post-instruction students tended to describe the eye as a single-lens system, composed of a lens and a screen (the retina). When asked about vision defects, less than half of the participants could explain near- and farsightedness. Of those who could accurately describe the defects, the vast majority indicated that they had corrective lenses for a vision defect. Most of the students felt that the problems resulted from a defect in the lens of the eye, and made no mention of the shape of the eye. In general, knowledge of the eye varied widely among students and did not seem to depend on previous instruction.

\section{Activated Resources}

When discussing the functions of the human eye and the wavefront aberrometer, the two groups of students used many of the same resources. Some of the common resources used by both pre- and postinstruction students follow, along with student quotes.

- The shape of a lens affects the image focus (11 of 19
students) "Well, this ... would change the diameter of the lens, which would determine how focused something would be."

- Lenses divide up the light (12 of 19 students) "then the [array] lenses are breaking up light ... and focusing it to their own point."

- Light entering a lens differently will focus differently (16 of 19 students)

"If you have a defect [in the lens], it's going to change the way that the light comes out of it, so it would have an effect on these lenses [of array] and how it comes out of them."

Beyond these resources, students who were postinstruction also had additional resources dealing with lenses, light, and the human eye. Listed here are three such resources that were particularly useful to students as they constructed an understanding of the aberrometer, but which were not exhibited by the preinstruction students. 
- The shape of the eye determines the focal point "You're looking at changing the distance, as far as the back of the eye ... it just goes hand-in-hand with the length of your eye. So if you move it [the retina] back, you're going to have to move this [the screen] either backward or forward, I just don't remember which."

\section{- Lenses redirect the light}

"Well, it looks like it's thicker through the middle. So that would be for the farsighted people - who can't see close." [Interviewer asks: "Okay, so what does the lens do?"] "It redirects the light. So the light goes through and it changes the focal point back here in our eyes."

- The distance light travels determines the angle "If it's a nearsighted eye, the dots are going to get closer together because the light is going to be coming in more parallel compared to... like, the angle between the top one and how far the light is away is going to be ... the further away the light is, the shallower the angle is.

Beyond these resources, another noticeable trend was the type of knowledge that students tended to rely on. For example, though one pre-instruction student was not familiar with the term "focal-point", he recalled that when he was young, he could light things on fire if he held a magnifying glass at the correct position. This type of 'experiential' knowledge was commonly discussed by the pre-instruction students.

The post-instruction students, however, frequently recited physics variables and equations. When discussing image formation in regards to the human eye, one student reasoned that:

"The $d_{0}$ changes, so the $d_{i}$ must change

since f stays the same."

This type of reasoning through physics equations (in particular the thin-lens equation) typically happened when students were discussing image and object placement, as well as when they were trying to decide how the radius-of-curvature of a convex lens would affect the focal point.

\section{Knowledge Construction Approach}

A notable difference between the pre- and postinstruction student groups arose when the wavefront aberrometer was first discussed. While the preinstruction students were willing to answer questions about the eye, they became very hesitant and reserved when the discussion switched to aberrometry. In fact, the learning/teaching interviews provided very little rich data because the pre-instruction students were hesitant to answer questions that pertained to the aberrometer. Many students simply answered "I don't know" or "I've never learned about this before," which might suggest that students were in an epistemic state in which knowledge is viewed as stuff that is propagated from authority [13].

Post-instruction students, on the other hand, were much more willing to discuss the physics of the wavefront aberrometer and showed much less hesitation in answering questions, making predictions, and postulating explanations for the phenomena occurring. This willingness to actively construct knowledge may result from the larger body of resources students seemed to have at their disposal as a result of traditional instruction. Another possible explanation is that their resources were betterorganized and therefore easily accessible during the knowledge construction process. This may also suggest that students were framing the situation as one in which knowledge could be constructed or freely created [13].

\section{Necessary Scaffolding}

By the end of the learning/teaching interview, all students, both pre- and post-instruction, had constructed an understanding of the wavefront aberrometer. However, very different levels of scaffolding were required for the pre- and postinstruction students.

The pre-instruction students required scaffolding in nearly every aspect of light, lenses, and the human eye. Scaffolding activities included exploration of converging and diverging lens (both hands-on and using a computer simulation) and manipulation of models of the human eye. Further, the pre-instruction students had to be encouraged to apply this newlylearned knowledge when they dealt with the wavefront aberrometer. Many hints, cues, and leading questions were necessary.

While the post-instruction students were asked to do the same activities as the pre-instruction students, they approached them as verifications of their prior knowledge. These students also readily applied their knowledge of basic optics and the human eye while constructing an understanding of wavefront aberrometry. Though some scaffolding was required, it was of much larger step-size than was required for the pre-instruction students, in that fewer details were required and the students were able to make larger cognitive leaps on their own.

An example of provided scaffolding was the drawing of light rays through a lens in order for them to think about what happened to the focal point. In 
many cases for the post-instruction students, this got them thinking about how the grid pattern would shift. However, this technique did not help the preinstruction students, as they had no prior knowledge about ray-diagrams or how to determine the focal point of the lens.

\section{CONCLUSIONS}

One important result is clear: students have a wide range of knowledge about the human eye, its functions, and vision defects. Students also have a significant body of resources that they use to understand aberrometry - some appropriately and some inappropriately.

It appears that the students who were preinstruction in light and basic optics were not prepared to construct an understanding of wavefront aberrometry without significant scaffolding. The students' hesitance with the material suggests that they did not realize that understanding wavefront aberrometry was within their range of capability - one might say that it was at the periphery of their Zone of Proximal Development (ZPD) [14]. Because they were able to activate the necessary resources during the process of understanding when we provided scaffolding, an understanding seemed to be within their ZPD.

Wavefront aberrometry was well within the ZPD of students who had some basic knowledge of light and optics, and they were more comfortable constructing knowledge in the new context. The post-instruction students were therefore able to construct their knowledge more independently and less scaffolding.

In many respects, students who had received instruction on the eye and lenses had a more predictable, though not flawless, understanding of the eye. However, it appears that while traditional instruction provided students with the confidence to use their knowledge of optics in this new context, it also may have hindered their use of some equallyproductive resources from everyday experiences. This is evidenced by their (sometimes incorrect) use of equations instead of resources they may have acquired elsewhere - resources that the pre-instruction students were readily able to use.

In general, students had many resources about the human eye and vision, many of which are artifacts of their everyday experiences. As would be expected, students have significantly fewer resources initially available to them about wavefront aberrometry. This study indicates that significant differences in level of preparedness and willingness to construct knowledge in a new context exist between students who are preor post- traditional instruction. However, this study also indicates that, despite these differences, all students are able to construct an understanding when appropriate scaffolding is provided.

\section{ACKNOWLEDGMENTS}

This work is supported by the National Science Foundation under grant DUE 0426754.

\section{REFERENCES}

1. Applegate, R.A., S. Marcos, and L.N. Thibos, Aberrometry: Clinical and research applications. Optometry and Vision Science, 2003. 80(2): p. 85-86.

2. Thibos, L.N., Principles of Hartmann-Shack Aberrometry. Journal of Refractive Surgery, 2000. 16(Sept/Oct): p. S563-S565.

3. Galili, I. and V. Lavrik, Flux concept in learning about light: A critique of the present situation. Science Education, 1998. 82(5): p. 591-613.

4. Saxena, A.B., The understanding of the properties of light by students in India. International Journal of Science Education, 1991. 13: p. 283-289.

5. Bendall, S., I. Galili, and F. Goldberg, Prospective elementary school teachers' prior knowledge about light. Journal of Research in Science Teaching, 1993. 30: p. 1169-1187.

6. Goldberg, F. and L.C. McDermott, Student difficulties in understanding image formation by a plane mirror. The Physics Teacher, 1986. 24: p. 472-480.

7. Goldberg and L.C. McDermott, An investigation of student understanding of the real image formed by a converging lens or concave mirror. American Journal of Physics, 1987. 55: p. 108-119.

8. Galili, I., S. Bendall, and F. Goldberg, The effects of prior knowledge and instruction on understanding image formation. Journal of Research in Science Teaching, 1993. 30: p. 271-301.

9. Engelhardt, P.V., et al. The teaching experiment - What it is and what it isn't. Physics Education Research Conference 2003.

10. Marton, F., Phenomenography—a research approach to investigating different understandings of reality. Journal of Thought, 1986. 21: p. 28-49.

11. McBride, D.L., Transfer of Learning from Basic Optics to Wavefront Aberrometry, in Department of Physics. 2009, Kansas State Univerisity: Manhattan, KS. p. 152.

12. Hammer, D., Student resources for learning introductory physics. American Journal of Physics Physics Education Research Supplement, 2000. 68(S1): p. S52-S59.

13. Hammer, D. and A. Elby, On the Form of a Personal Epistemology, in Personal Epistemolgy: The Psychology of Beliefs about Knowledge and Knowing, B.K. Hofer and P.R. Pintrich, Editors. 2002, Erlbaum: Mahwah, NJ. p. 169-190.

14. Vygotsky, L.S., Mind in Society: The developemnt of Higher Psychological Processes. 1978, Cambridge: Harvard University Press. 